\title{
Efficacy and tolerability of oral oxycodone and oxycodone/naloxone combination in opioid-naïve cancer patients: a propensity analysis
}

This article was published in the following Dove Press journal:

Drug Design, Development and Therapy

2 November 2015

Number of times this article has been viewed

\author{
Marzia Lazzari' \\ Maria Teresa Greco ${ }^{2,3}$ \\ Claudio Marcassa ${ }^{4}$ \\ Simona Finocchi' \\ Clarissa Caldarulo' \\ Oscar Corli \\ 'Emergency Care, Critical Care \\ Medicine, Pain Medicine and \\ Anesthesiology Department, Tor \\ Vergata Polyclinic, University of Rome, \\ Rome, ${ }^{2}$ Oncology Department, Pain \\ and Palliative Care Research Unit, \\ Mario Negri IRCCS, ${ }^{3}$ Department \\ of Clinical Sciences and Community, \\ University of Milan, Milan, ${ }^{4}$ Cardiology, \\ Fondazione Maugeri IRCCS Veruno, \\ Novara, Italy
}

Correspondence: Marzia Lazzari Tor Vergata Polyclinic, University of Rome "Tor Vergata", Viale Oxford 8I, 00133 Rome, Italy

Tel +3906 20900638

$\mathrm{Fax}+390620904846$

Email marzialaz@hotmail.it
Background: World Health Organization step III opioids are required to relieve moderate-to-severe cancer pain; constipation is one of the most frequent opioid-induced side effects. A fixed combination, prolonged-release oxycodone/naloxone (OXN), was developed with the aim of reducing opioid-related gastrointestinal side effects. The objective of this study was to compare the efficacy and safety of prolonged-release oxycodone (OXY) alone to OXN in opioid-naïve cancer patients with moderate-to-severe pain.

Methods: Propensity analysis was utilized in this observational study, which evaluated the efficacy, safety, and quality of life.

Results: Out of the 210 patients recruited, 146 were matched using propensity scores and included in the comparative analysis. In both groups, pain intensity decreased by $\approx 3$ points after 60 days, indicating comparable analgesic efficacy. Responder rates were similar between groups. Analgesia was achieved and maintained with similarly low and stable dosages over time (12.0-20.4 mg/d for OXY and 11.5-22.0 mg/d for OXN). Bowel Function Index (BFI) and laxative use per week improved from baseline at 30 days and 60 days in OXN recipients $(-16, P<0.0001$ and $-3.5, P=0.02$, respectively); BFI worsened in the OXY group. The overall incidence of drug-related adverse events was $28.9 \%$ in the OXY group and $8.2 \%$ in the OXN group $(P<0.01)$; nausea and vomiting were two to five times less frequent with OXN. Quality of life improved to a significantly greater extent in patients receiving OXN compared to OXY (increase in Short Form-36 physical component score of 7.1 points vs 3.2 points, respectively; $P<0.001)$.

Conclusion: In patients with chronic cancer pain, OXN provided analgesic effectiveness that is similar to OXY, with early and sustained benefits in tolerability. The relationship between responsiveness to $\mathrm{OXN}$ and clinical characteristics is currently being investigated.

Keywords: cancer pain, analgesia, oxycodone, naloxone, propensity analysis

\section{Background}

World Health Organization (WHO) guidelines for pharmacological management of cancer pain consider that opioid analgesics are an established therapy for moderate-tosevere cancer pain. ${ }^{1}$ The European Association of Palliative Care (EAPC) recommends the use of morphine, oxycodone (OXY), and hydromorphone as first-line options for therapy with a strong opioid., ${ }^{2,3}$ In a recent study assessing the effectiveness of four strong opioids (morphine, buprenorphine, OXY, and fentanyl) in cancer patients, the efficacy and tolerability profiles of these drugs were found to be broadly similar, although some variability in response among different opioids was seen. ${ }^{4}$ In particular, a series of systematic reviews on the use of opioids in cancer pain reported that OXY, 
a semisynthetic opioid analgesic, has an efficacy and tolerability that are similar to morphine.,

The clinical use of opioids is limited by the occurrence of problematic adverse effects. The most common adverse event is opioid-induced bowel dysfunction, which includes constipation, hard dry stools, incomplete evacuation, bloating, abdominal cramping, and increased gastric reflux. Bowel dysfunction occurs in $\sim 90 \%$ of cancer patients receiving opioid therapy $;^{7}$ the frequency of the above-mentioned symptoms ranges from $26 \%$ to $49 \%$ in patients receiving OXY. ${ }^{8-11}$ Both the European Society of Medical Oncology and the EAPC recommend routine use of laxatives in patients with advanced cancer receiving opioids. ${ }^{2,12}$

A fixed combination of oral prolonged-release oxycodone and naloxone $(\mathrm{OXN})$ in a 2:1 ratio has been developed in the attempt to minimize the adverse gastrointestinal effects of opioids. ${ }^{13}$ Naloxone is an opioid receptor antagonist with very low bioavailability ( $<3 \%$ ) following oral administration as it undergoes extensive hepatic first-pass metabolism. ${ }^{14}$ Moreover, intestinal binding of OXY to opioid receptors is inhibited by naloxone, due to the higher receptor affinity for the latter. ${ }^{13}$ On the other hand, the low plasma levels of naloxone do not interfere with the central analgesic action of OXY. ${ }^{13}$

The effectiveness and tolerability of $\mathrm{OXN}$ in patients with noncancer pain have been previously studied. ${ }^{15-17}$ In addition, three randomized clinical trials (RCTs) have confirmed that $\mathrm{OXN}$ has analgesic efficacy that is similar to OXY but with significant improvement in opioid-induced constipation (OIC). ${ }^{18-20}$ A few observational studies and one RCT have assessed the efficacy and tolerability of OXN in cancer pain. ${ }^{21-25}$ In particular, the RCT showed that OXN was associated with fewer adverse effects related to bowel function compared with OXY alone, without significant differences in analgesic efficacy. ${ }^{24}$ However, this trial exclusively enrolled opioid-pretreated patients with controlled pain suffering from OIC. Data from RCTs comparing the efficacy and tolerability of OXN vs OXY in cancer patients who are naïve to opioids and with uncontrolled pain are lacking.

Herein, we employed propensity analysis, an alternative statistical method that allows comparison of data from noninterventional observational studies, which has already been used in this clinical context. ${ }^{26}$ Propensity analysis adjusts for bias inherent to the decision about opioid therapy, balancing the variables related to the choice of exposure (treatment), in order to simulate the random assignment. ${ }^{27}$ The aim of the present study was to compare the effects of OXY and $\mathrm{OXN}$ on analgesic efficacy, safety, and quality of life (QoL) in opioid-naïve patients with moderate-to-severe chronic cancer pain.

\section{Methods}

\section{Study design, patients, and assessment}

This was a single-center, observational, retrospective study assessing patients with cancer treated at the Tor Vergata Polyclinic Pain Unit in Rome, Italy, between January 2010 and December 2012. The study was approved by the University of Tor Vergata Review Board (registry number 175/13). Informed consent was exempted by the board due to the retrospective nature of this research.

Patients were eligible for inclusion in the study if they met the following criteria: diagnosis of solid tumor, the presence of moderate-to-severe pain at baseline defined as average pain intensity (API) score $\geq 4$ measured on a $0-10$ numerical rating scale and requiring around-the-clock WHO step III opioids (strong opioids), no previous treatment with around-the-clock opioids (ie, opioid naïve), and suitable for treatment with a new prescription of prolonged-release OXY or OXN. Pregnant women, patients with a history of alcohol and/or drug abuse or cognitive impairment, and those treated with chemo- and/or radiotherapy in the 15 days before or during the observation period were excluded.

Patients were evaluated at baseline (T0) and at 30 days (T30) and 60 days (T60: final observation). An optional visit for patients requiring closer monitoring and titration of analgesic treatment was scheduled after 15 days (T15). Demographic information and clinical history collected at baseline included age, sex, primary tumor site, the presence and sites of metastases, previous treatments for pain, the presence and severity of neuropathic pain (NP), and laxative use. The starting dose of OXY $(5-20 \mathrm{mg} / \mathrm{d})$ or OXN $(5 / 2.5-20 / 10 \mathrm{mg} / \mathrm{d})$ was determined on an individual basis by a pain management specialist according to patient needs and previous analgesic therapy.

\section{Effectiveness endpoints}

API was determined at each visit. Patients with an API difference (APID) $\geq 30 \%$ and a final API score $\leq 4$ were defined as "responders"., 4,28,29 NP was assessed using the Douleur Neuropathique 4 (DN4) questionnaire, which consists of pain descriptors and several bedside sensory examinations. ${ }^{30,31}$ The absolute daily dose of OXY, either alone or in combination with naloxone, and the proportion of patients with an increase of $\geq 5 \%$ per day in the OXY dose were determined during the observation period according to the Opioid Escalation Index (OEI) percentage. ${ }^{32}$

\section{QoL endpoints}

QoL endpoints were evaluated at baseline and T60. The presence and severity of sleep disturbance were evalu- 
ated using the Chronic Pain Sleep Inventory (CPSI), a single index assessing five parameters of sleep troubles related to pain (trouble falling asleep, needing sleep medication, awakened by pain during the night and in the morning, and overall quality of sleep) on a $100 \mathrm{~mm}$ visual analog scale. ${ }^{33}$ Physical and mental well-being were assessed using the Physical (PCS) and Mental Component Summary (MCS) scores of the Short Form-12 Health Survey Questionnaire (SF12); higher scores reflect better QoL. ${ }^{34,35}$

\section{Tolerability and safety endpoints}

Bowel function was measured at T30 and T60 using the Bowel Function Index (BFI) questionnaire. ${ }^{36}$ The BFI is a measure of general bowel function that has been recently validated as a reproducible tool to detect clinically meaningful changes in OIC, with scores ranging from 0 (free from symptoms) to 100 (most severe symptoms). In patients with chronic pain, normal bowel function is defined as a BFI $\leq 29$, and $\mathrm{a} \geq 12$-point change in BFI score represents a clinically meaningful change in severity of constipation. ${ }^{37}$ The proportion of patients receiving laxatives and the number of laxatives taken per week were also determined at baseline, T30, and T60.

Safety evaluations were performed at each visit by recording adverse drug reactions (ADRs), defined as any new side effect occurring or worsening in intensity and/or frequency after the first intake of OXY or OXN. Only ADRs of moderate or severe intensity were recorded.

A composite efficacy and tolerability endpoint was used to evaluate the overall risk:benefit profile of OXY and OXN; this included the simultaneous presence of APID $\geq 30 \%$ and $\mathrm{BFI}<51$ points at $\mathrm{T} 60$, which indicates a substantial analgesic benefit in the absence of moderate-to-severe bowel dysfunction.

\section{Statistical analysis}

In the descriptive analysis, absolute frequency was used for categorical variables, and central trend and dispersion measurements (mean, standard deviations) for quantitative continuous variables. Normality of data distribution was verified by the Shapiro-Wilk test.

Propensity analysis aims to identify patients with similar probability of receiving one of the two treatments on the basis of observed clinical characteristics. ${ }^{27,38,39}$ A propensity score model was generated using all potential covariates that could affect the group allocation using a multivariable logistic regression model which includes several characteristics as independent variables to estimate the individual probability of being assigned to OXY or OXN. If two patients have the same propensity score, they share the same probability of receiving one of two treatments. The variables included in the propensity score model included age, sex, the presence of metastasis, NP, OIC, API, laxative use, sleep disturbance, and physical and mental well-being at baseline. One-to-one matching without replacement was performed using a 0.2 caliper width, and the resulting score-matched pairs were used in subsequent analyses.

In the event of early discontinuation or missing values, the last-observation-carried-forward approach was used to input missing data on API, OXY dosage, DN4, CPSI, BFI, laxative use, and MCS and PCS scores of SF12.

When comparing groups, chi-square or Fisher's exact test for associations was used for categorical variables. For continuous or ordinal variables, the Kolmogorov-Smirnov test was applied. Changes over time were evaluated with the Cochran Q test, Friedman test, or repeated measures analysis of variance as appropriate. A $P$-value of $<0.05$ was considered statistically significant. STATISTICA software (Version 10.0; StatSoft Inc., Tulsa, OK, USA) was used for all statistical analyses. Propensity matching was performed with the MatchIt Package. ${ }^{40}$

\section{Results}

A total of 210 consecutive patients treated with OXY or OXN for moderate-to-severe cancer pain were evaluated. Following propensity score analysis, 73 patients receiving OXY and 73 receiving OXN were matched. Over the 60-day observation period, 15 patients $(10.2 \%)$ withdrew from treatment (all due to ADRs): four in the OXN group and eleven in the OXY group. The remaining 131 patients $(89.8 \%)$ continued with their new analgesic treatment up to the end of the observation period. Patient flow throughout the study is shown in Figure 1.

There were no significant differences between the propensity score-matched groups at baseline (Table 1), indicating that the matching procedure worked well. The most common sites of cancer were pulmonary, breast, and genitourinary. API was 7.3 in both groups, and there was a high prevalence of NP $(>77 \%)$. The BFI score at baseline suggested the absence of overt bowel dysfunction prior to opioid prescription, although one-quarter of patients were already on laxatives.

\section{Analgesic effectiveness and QoL}

API decreased markedly from baseline to T60 (Figure 2) from $7.3 \pm 1.6$ to $4.2 \pm 2.3$ in OXY recipients and from $7.3 \pm 1.4$ to $4.3 \pm 1.7$ in $\mathrm{OXN}$ recipients ( $P$, not significant). The APID 


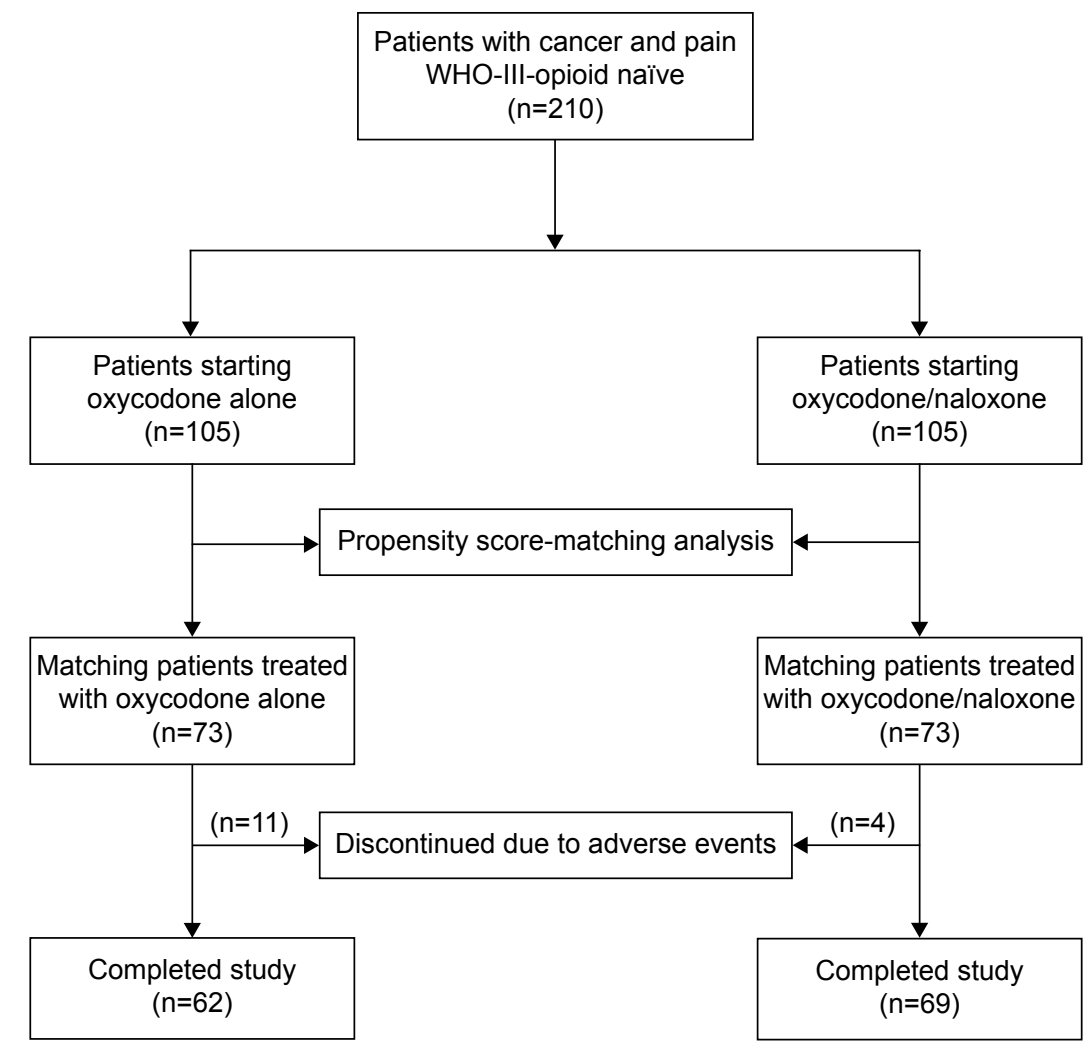

Figure I Patient flow during the study.

Abbreviation: WHO, World Health Organization.

from baseline to T60 was $3.1 \pm 2.3$ and $3.0 \pm 1.8$ in the $\mathrm{OXY}$ and OXN groups, respectively ( $P$, not significant). The responder rate was $50.7 \%$ in both groups.

NP also significantly decreased in both the OXY and OXN groups $(P<0.001)$. Average DN4 scores decreased over 60 days of treatment, from $5.0 \pm 1.2$ to $3.3 \pm 1.7$ in the OXY group and from $4.7 \pm 1.4$ to $2.7 \pm 2.2$ in the OXN group. The proportion of patients with DN4 $\geq 4$ also decreased significantly in both groups at T60 $(52.8 \%$ of those treated with OXY and $43.8 \%$ of OXN recipients; both $P<0.001$ vs baseline, no significant between-group difference). Changes in OXY dosages over time were similar in the two treatment groups (Figure 3): the daily dosages of OXY increased similarly and slightly in both groups (by $<10 \mathrm{mg}$ after 30 days and by $<15 \mathrm{mg}$ after 60 days). The prevalence of patients with OEI $>5 \%$ at 60 days was low and comparable between groups $(2.7 \%$ and $1.4 \%$ in OXY and OXN recipients, respectively).

There were also no significant differences between the OXY and OXN groups in QoL assessments during the study (Table 2), with the exception of the SF12 PCS score, which increased by 7.0 points in the $\mathrm{OXN}$ group and 3.2 points in the OXY group $(P<0.001)$.

\section{Tolerability and safety}

Considering gastrointestinal tolerability (Table 3 ), early improvement in BFI was seen in OXN recipients $(-16$ points), while BFI worsened in OXY recipients $(+13.8$ points; between-group difference, $P<0.001$ ). The differences in BFI variations were above the clinically meaningful threshold ( \pm 12 points) in both groups, indicating clinically relevant changes in the severity of constipation. Laxative use decreased from baseline to T60 in the OXN group, and increased in the OXY group, with significant differences between groups (Table 3).

Eleven OXY recipients (15.0\%) reported severe ADRs (constipation, $\mathrm{n}=5$; vomiting, $\mathrm{n}=2$; drowsiness, headache, insomnia, and pruritus in one patient each) that led to early discontinuation of analgesic treatment. In the OXN group, four OXN recipients $(5.5 \%)$ discontinued early due to intolerable ADRs (vomiting, $\mathrm{n}=2$; dizziness and drowsiness in one patient each). In the remaining patients who completed follow-up, the overall rate of moderate-to-severe ADRs was $18.4 \%$ (28.9\% OXY and 8.2\% OXN; $P=0.002$ ) (Table 4). Nausea and vomiting were significantly more frequent with OXY than with OXN (overall $17.8 \%$ vs $5.4 \%$, respectively; $P<0.05$ ) (Table 4). 
Table I Baseline characteristics of the study population

\begin{tabular}{|c|c|c|c|c|}
\hline & Total $(n=\mid 46)$ & Oxycodone (n=73) & Oxycodone + naloxone $(n=73)$ & $P$-value \\
\hline Age (years) & $62.1 \pm 13.2$ & $63.3 \pm 10.3$ & $62.7 \pm 15.3$ & NS \\
\hline Male, n (\%) & $76(52.1)$ & $35(47.9)$ & $4 \mid(56.2)$ & NS \\
\hline \multicolumn{5}{|l|}{ Primary tumor site, $\mathrm{n}(\%)$} \\
\hline Pulmonary & $28(19.2)$ & $12(16.4)$ & $16(21.9)$ & NS \\
\hline Breast & $18(12.3)$ & II (I5.I) & $7(9.6)$ & NS \\
\hline Prostate & $12(8.2)$ & $7(9.6)$ & $5(6.8)$ & NS \\
\hline Genitourinary & $12(8.2)$ & $4(5.5)$ & $8(10.9)$ & NS \\
\hline Head and neck & $12(8.2)$ & $6(8.2)$ & $6(8.2)$ & NS \\
\hline Pancreas & II (7.5) & $5(6.8)$ & $6(8.2)$ & NS \\
\hline Myeloma & II (7.5) & $6(8.2)$ & $5(6.8)$ & NS \\
\hline Lymphoma & $9(6.2)$ & $3(4.1)$ & $6(8.2)$ & NS \\
\hline Gynecologic & $8(5.6)$ & $7(9.6)$ & $\mathrm{I}(\mathrm{l} .7)$ & NS \\
\hline Gastrointestinal & $7(4.8)$ & $5(6.8)$ & $2(2.7)$ & NS \\
\hline Liver & $4(2.8)$ & $2(2.8)$ & $2(2.7)$ & NS \\
\hline Others & $14(9.6)$ & $5(6.8)$ & $9(12.3)$ & NS \\
\hline Bone metastasis, n (\%) & $39(26.7)$ & $24(32.9)$ & $15(20.5)$ & 0.17 \\
\hline Pain intensity, NRS score & $7.3 \pm 1.5$ & $7.3 \pm 1.6$ & $7.3 \pm 1.4$ & NS \\
\hline Neuropathic pain*, n (\%) & II $3(77.4)$ & $61(83.6)$ & $52(7 \mid .2)$ & 0.08 \\
\hline DN4 inventory & $4.9 \pm 1.4$ & $4.7 \pm 1.4$ & $5.0 \pm 1.2$ & NS \\
\hline \multicolumn{5}{|c|}{ Previous WHO primary analgesics, n (\%) } \\
\hline None & $77(52.7)$ & $28(38.3)$ & $49(67.1)$ & 0.001 \\
\hline NSAIDs & $48(32.8)$ & $32(43.8)$ & $16(21.9)$ & $<0.05$ \\
\hline Paracetamol & $21(14.3)$ & $13(17.8)$ & $8(10.9)$ & NS \\
\hline Rescue pain medication, n (\%) & $20(13.7)$ & $12(16.4)$ & $8(11.0)$ & NS \\
\hline Paracetamol** & $14(9.6)$ & $9(12.3)$ & $5(6.8)$ & \\
\hline Opioids $* * *$ & $6(4.1)$ & $3(4.1)$ & $3(4.1)$ & \\
\hline \multicolumn{5}{|l|}{ Adjuvant pain medication } \\
\hline Pregabalin, n (\%) & $95(65.1)$ & $47(64.4)$ & $48(65.8)$ & NS \\
\hline Pregabalin dose $(\mathrm{mg} / \mathrm{d})$ & $27.2 \pm 6.4$ & $27.1 \pm 6.8$ & $27.5 \pm 6.3$ & NS \\
\hline Other drugs, n (\%) & $15(10.3)$ & II (I5.0) & $4(5.4)$ & NS \\
\hline CPSI score & $33.3 \pm 14.4$ & $33.1 \pm 16.2$ & $33.2 \pm 12.6$ & NS \\
\hline SFI2 MCS score & $27.2 \pm 6.4$ & $27.1 \pm 6.8$ & $27.5 \pm 6.3$ & NS \\
\hline SFI2 PCS score & $30.5 \pm 5.2$ & $30.4 \pm 4.9$ & $30.6 \pm 5.5$ & NS \\
\hline BFI score & $26.5 \pm 26.8$ & $27.1 \pm 25.8$ & $25.5 \pm 27.8$ & NS \\
\hline $\mathrm{BFI}>29, \mathrm{n}(\%)$ & $73(50.0)$ & $38(52.1)$ & $35(47.9)$ & NS \\
\hline Laxative use, $\mathrm{n}(\%)$ & $4 I(28.1)$ & $21(28.8)$ & $20(27.4)$ & NS \\
\hline Oxycodone starting dose $(\mathrm{mg} / \mathrm{d})$ & $11.7 \pm 4.3$ & $12.0 \pm 4.4$ & II. $5 \pm 4.3$ & NS \\
\hline
\end{tabular}

Notes: Values are mean \pm standard deviation or number of patients (\%). *Includes neuropathic and mixed nociceptive-neuropathic pain. $* *$ Paracetamol I,000 mg. *** Immediate-release oral morphine $10 \mathrm{mg}$ or transmucosal fentanyl $100 \mu \mathrm{g}$.

Abbreviations: BFI, Bowel Function Index; CPSI, Chronic Pain Sleep Inventory; DN4, Douleur Neuropathique 4; NRS, numerical rating scale; NS, not significant; NSAIDs, nonsteroidal anti-inflammatory drugs; SFI2 MCS, Mental Component Summary of the Short Form- 2 Health Survey Questionnaire; SFI2 PCS, Physical Component Summary of the Short Form- 12 Health Survey Questionnaire; WHO, World Health Organization.

\section{Composite endpoint}

After 60 days of observation, 31 patients $(42.5 \%)$ treated with OXY and 47 (64.4\%) treated with OXN achieved the composite efficacy and tolerability endpoint (ie, APID $\geq 30 \%$ and final BFI $<51)(P=0.01)$.

\section{Discussion}

This study utilized propensity analysis to study a sample of consecutive cancer patients with moderate-to-severe pain intensity $\geq 4$ points in order to compare the efficacy, safety, and QoL of prolonged-release OXY and OXN, two of the most widely prescribed opioids.
Over 60 days of treatment, there was a decrease of $\sim 3$ points in the API in both groups. In addition, the prevalence of NP decreased from baseline by $30 \%$. These findings are in agreement with data from previous RCTs showing that OXY and OXN have equivalent analgesic effects in both chronic cancer pain and noncancer pain. ${ }^{18,20,24,42}$ In contrast, an open-label, randomized trial comparing morphine, $\mathrm{OXY}$, and $\mathrm{OXN}$ in 453 patients requiring WHO step III opioids to treat lower back pain showed that OXN had significantly better analgesic efficacy and tolerability, with a lower risk of discontinuation. ${ }^{41}$

In our study, the proportion of responders was also the same following treatment with either OXY or OXN $(50.7 \%$ 


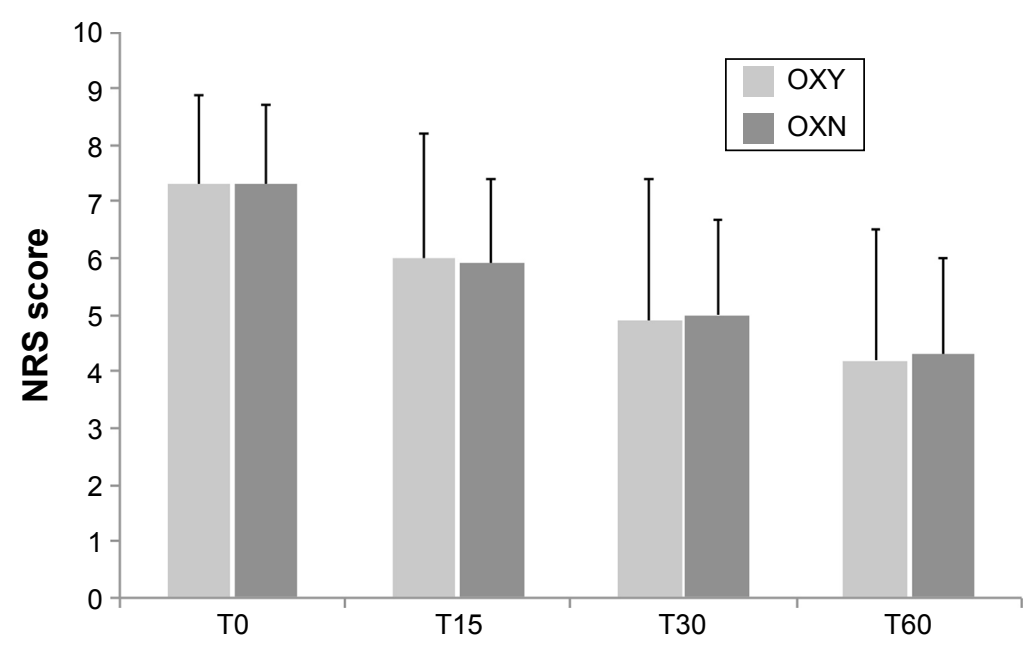

Figure 2 Average pain intensity (score on an II-point NRS) during treatment with OXY and OXN.

Notes: $P$-values: within groups over time, $<0.00 \mathrm{I}$; between groups, 0.90 ; interaction, 0.91 . $\mathrm{T} 0=$ baseline, $\mathrm{TI}=\mathrm{fay} 15, \mathrm{~T} 30=$ day 30 , and $\mathrm{T} 60=$ day 60 .

Abbreviations: NRS, numerical rating scale; OXN, oxycodone/naloxone; OXY, oxycodone.

in both groups). The concept of "responder" provides different information about the analgesic response to therapy depending on how response is defined. In clinical studies, the results are mainly driven by mean values for specified outcomes and endpoints. However, this does not allow identification of good and poor responses to treatment. In this study, responders were defined as those who reported a decrease in the API score $\geq 30 \%$ from baseline at T60, and an absolute API score $\leq 4$ at the final visit. ${ }^{29,42-46}$

Approximately half of the patients in this study were classified as responders; conversely, about half of patients did not satisfy the predefined analgesic effectiveness criteria, a finding consistent with previous reports. ${ }^{4,46}$ This proportion of nonresponders may reflect the large number of patients who are not or poorly susceptible to OXY, or more in general to opioid analgesics, which are still considered to be the most effective agents for treatment of moderate-to-severe cancer pain. On the other hand, the low OXY dosages prescribed in the first 2 months in the current study population could partially explain the nonresponse rate. The modest increases in OXY dosage over time showed a stable ratio between dose and pain relief: the average final dose was $20.4 \mathrm{mg} / \mathrm{d}$ in the OXY group and $22.0 \mathrm{mg} / \mathrm{d}$ in the OXN group. Dose escalation during opioid therapy is an indicator of development of tolerance, and the slow increase in dosages observed in this study is thus a favorable indicator. Nevertheless, the causes of and alternative solutions to the limited effectiveness of opioids in cancer pain require further investigation.

The relatively low opioid doses used in this study may be one explanation for the good safety profile observed.

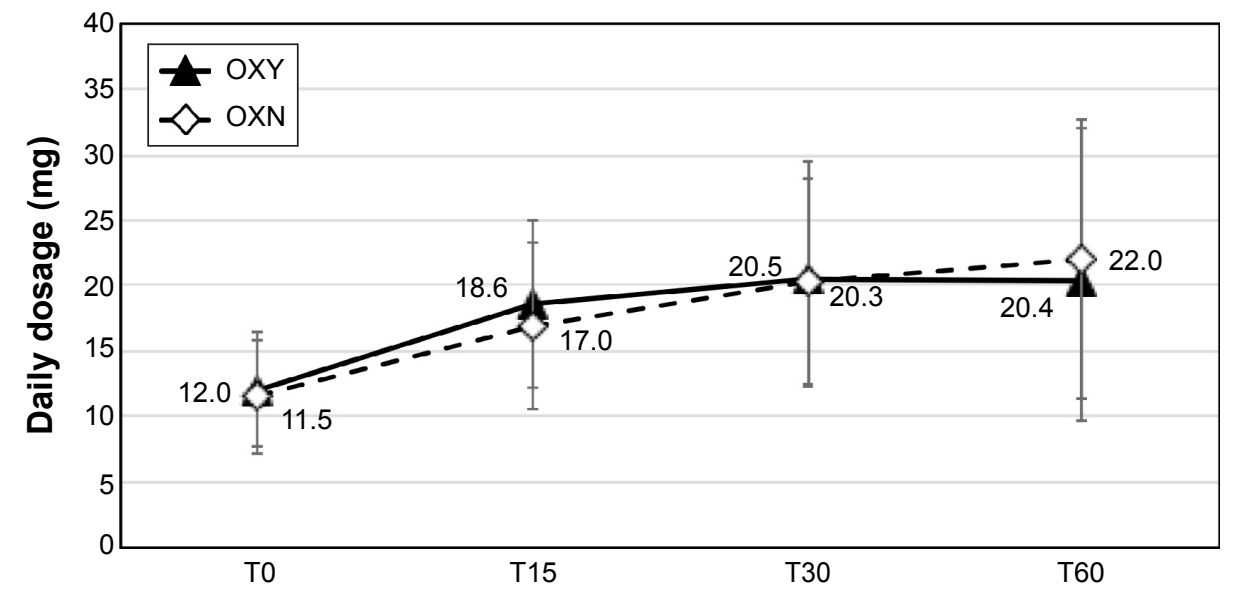

Figure $3 \mathrm{OXY}$ and $\mathrm{OXN}$ mean daily dosages during the study.

Note: $\mathrm{T} 0=$ baseline, $\mathrm{TI} 5=$ day $15, \mathrm{~T} 30=$ day 30 , and $\mathrm{T} 60=$ day 60 .

Abbreviations: OXN, oxycodone/naloxone; OXY, oxycodone. 
Table 2 Summary of quality of life endpoints and comparison between groups

\begin{tabular}{llll}
\hline & Oxycodone $(\mathbf{n}=\mathbf{7 3})$ & Oxycodone + naloxone $(\mathbf{n}=\mathbf{7 3})$ & $\mathbf{P}$-value \\
\hline CPSI score & & & \\
$\quad$ Baseline & $33.1 \pm 16.2$ & $33.2 \pm 12.6$ & $\mathrm{NS}$ \\
Day 60 & $57.1 \pm 17.6$ & $60.3 \pm 16.5$ & $\mathrm{NS}$ \\
Difference & $24.0 \pm 22.4$ & $27.2 \pm 15.5$ & $\mathrm{NS}$ \\
SFI2 MCS score & & & $\mathrm{NS}$ \\
Baseline & $27.1 \pm 6.8$ & $27.4 \pm 6.4$ & 0.01 \\
Day 60 & $37.4 \pm 8.3$ & $38.3 \pm 5.1$ & $\mathrm{NS}$ \\
Difference & $10.3 \pm 8.9$ & $10.9 \pm 7.0$ & $\mathrm{NS}$ \\
SFI2 PCS score & & & $<0.001$ \\
Baseline & $30.4 \pm 4.9$ & $30.6 \pm 5.5$ & $<0.001$ \\
Day 60 & $33.6 \pm 4.5$ & $37.6 \pm 5.0$ & \\
Difference & $3.2 \pm 6.1$ & $7.1 \pm 5.7$ & \\
\hline
\end{tabular}

Note: Values are mean \pm standard deviation.

Abbreviations: CPSI, Chronic Pain Sleep Inventory; NS, not significant; SFI2 MCS, Mental Component Summary of the Short Form- 12 Health Survey Questionnaire; SFI2 PCS, Physical Component Summary of the Short Form- 12 Health Survey Questionnaire.

ADRs typically associated with opioid therapy were generally infrequent but were significantly more frequent with OXY that with OXN; in particular, nausea and vomiting were two to five times more common with OXY than with OXN. A possible explanation for this difference is that naloxone may act at the gastric level via the same mechanism involved in reducing constipation. A lower rate of nausea and vomiting during $\mathrm{OXN}$ therapy has also been noted in a previous report. ${ }^{23}$

The issue of constipation requires special attention. Opioids cause or worsen constipation by binding $\mu$-opioid receptors at the intestinal level, decreasing gastric emptying and intestinal peristalsis. In this study, the degree of constipation, measured by BFI, and the proportion of patients with moderate-to-severe bowel dysfunction $(\mathrm{BFI}>50)$ at baseline were similar in the OXY and OXN groups but significantly diverged during the study; bowel function worsened with OXY therapy but improved with OXN, as also suggested by differences in laxative use between groups.

This study utilized a composite endpoint to fully assess the benefit of OXY and OXN. A combined efficacy-tolerability endpoint (in our study, the proportion of patients with $\geq 30 \%$ reduction in pain intensity and a BFI score $<51$ ) is rather unusual but in our opinion summarizes well the two main

Table 3 Summary of intestinal function endpoints and comparison between groups

\begin{tabular}{|c|c|c|c|}
\hline & Oxycodone $(n=73)$ & Oxycodone + naloxone $(n=73)$ & $P$-value \\
\hline \multicolumn{4}{|l|}{ BFI score } \\
\hline Baseline & $27.1 \pm 25.8$ & $25.5 \pm 27.8$ & \multirow[t]{3}{*}{ NS } \\
\hline Day 30 & $40.3 \pm 29.3$ & $12.1 \pm 16.5$ & \\
\hline Day 60 & $41.0 \pm 27.5$ & $9.5 \pm 13.4$ & \\
\hline Baseline to day 30 difference & $13.2 \pm 17.9$ & $-13.4 \pm 16.7$ & $<0.001$ \\
\hline Baseline to day 60 difference & $13.8 \pm 19.7$ & $-16.0 \pm 19.2$ & $<0.001$ \\
\hline \multicolumn{4}{|l|}{ Laxative use, $\%$ of patients } \\
\hline Baseline & 28.8 & 21.9 & \multirow[t]{3}{*}{ NS } \\
\hline Day 30 & 26.0 & 17.8 & \\
\hline Day 60 & 24.6 & 8.2 & \\
\hline Baseline to day 30 difference & -2.8 & -4.1 & NS \\
\hline Baseline to day 60 difference & -4.2 & -13.7 & 0.08 \\
\hline \multicolumn{4}{|l|}{ Laxative use, times per week } \\
\hline Baseline & $4.5 \pm 1.4$ & $4.3 \pm 1.9$ & \multirow[t]{3}{*}{ NS } \\
\hline Day 30 & $5.5 \pm 1.6$ & $1.8 \pm 0.8$ & \\
\hline Day 60 & $5.4 \pm 1.7$ & $1.2 \pm 0.4$ & \\
\hline Baseline to day 30 difference & $0.5 \pm 1.4$ & $-2.2 \pm 1.7$ & $<0.001$ \\
\hline Baseline to day 60 difference & $0.7 \pm 1.8$ & $-3.5 \pm 2.4$ & $<0.001$ \\
\hline
\end{tabular}

Note: Values are mean \pm standard deviation, or percentage of patients.

Abbreviations: BFI, Bowel Function Index; NS, not significant. 
Table 4 Summary of moderate-to-severe adverse drug reactions

\begin{tabular}{llll}
\hline & Oxycodone (n=73) & Oxycodone + naloxone (n=73) & P-value \\
\hline Nausea & $9(12.3)$ & $2(2.7)$ & 0.06 \\
Vomiting & $4(5.5)$ & $2(2.7)$ & NS \\
Abdominal pain & $1(1.4)$ & 0 & NS \\
Dizziness & $1(1.4)$ & 0 & NS \\
Sleepiness & $3(4.1)$ & $1(1.4)$ & NS \\
Headache & $I(1.4)$ & 0 & NS \\
Asthenia & 0 & $1(1.4)$ & NS \\
Insomnia & $I(1.4)$ & 0 & NS \\
Itch & $I(1.4)$ & 0 & NS \\
Total & $21(28.9)$ & $6(8.2)$ & 0.002 \\
\hline
\end{tabular}

Note: Values are number of patients (\%).

Abbreviation: NS, not significant.

goals of effective treatment for cancer pain, namely achieving good analgesia and avoiding or reducing the severity of typical opioid-related side effects, such as OIC. The better performance of OXN on a combined endpoint can thus be considered to be a good overall summary of the study findings.

The results of our propensity analysis are concordant with the results of other studies assessing efficacy and tolerability of OXN in cancer patients. ${ }^{21-25}$ In a retrospective study of 206 ambulatory patients with uncontrolled pain, Cuomo et al found that OXN was highly effective and without adverse effects on bowel function, and equally efficacious and well tolerated in both opioid-naïve and experienced patients, as well as in younger and older patients. ${ }^{21}$ In that study, however, patients previously treated with OXY were not included. A small nonrandomized, open-label study documented clinically relevant improvement in pain intensity and bowel function, as well as increased patient satisfaction, after treatment with OXN. ${ }^{22}$ A previous RCT compared the efficacy and safety of OXY and OXN in a very selected cohort of opioid-pretreated cancer patients with controlled pain and OIC at baseline: patients who were switched from other opioids to OXN experienced a similar analgesic effect as well as clinically relevant improvement in bowel function compared with patients switched to OXY. ${ }^{24}$ However, additional randomized studies are needed to compare different opioids in unselected patients with cancer pain.

This study has several limitations related to the methodology used to control for the unbalance between groups, which should be taken into account when judging the internal and external validity of the results. First, the assumption that sufficient variance has been considered in the propensity model should be effectively demonstrated. To optimize the propensity model, data collection was based on the current literature ${ }^{10-17}$ and expert recommendations, ${ }^{38,39}$ and most of the relevant variables were included in the propensity model.
Unlike randomization, propensity analysis can only remove overt (known) bias; however, hidden (unmeasured) biases remain, and therefore, the results generated using propensity matching should be interpreted with caution. The validity of the results will depend on the quality and amount of information on the efficacy and safety of the treatments under evaluation. In addition, the characteristics of the sample population are also important: propensity analysis allows limited extrapolations to different patient groups and settings with a distribution of covariates that differs from the one used for score generation. For instance, our population showed a high (77.4\%) prevalence of NP at study entry, which is a rather distinctive finding. The high rate of NP found in our study may be related to the systematic screening with the DN4 at baseline and the inclusion of mixed nociceptive pain and NP; a similar rate has also been recently reported. ${ }^{21}$ The problem of external validity (generalizability) of the results is consistent, and other relevant determinants involved in physician and patient decisions about whether or not to prescribe and use a treatment are often not fully considered.

Despite these cautions and inherent limitations, use of a propensity model may improve understanding of the actual value of the results from observational studies.

Despite the above-mentioned limitations and the singlecenter nature of this study, the patient population is nonetheless representative of those with moderate-to-severe cancer pain requiring treatment with WHO step III opioids, and may provide useful guidance for daily management of these patients.

\section{Conclusion}

The results of this study confirm previous data, suggesting that the analgesic properties of OXN are similar to OXY, with less OIC, and extend these findings to patients with cancer pain. In fact, effective analgesia was documented using low 
and stable dosages, in addition to a good safety profile and a reduced prevalence of nausea and vomiting with OXN vs OXY. In addition, these results provide important information about the proportion of patients achieving good or inadequate overall responses to analgesic therapy. This is applicable to all opioids and suggests the need to investigate the causes of opioid noneffectiveness and strategies to overcome this problem. Areas for future research include how the response to opioids is related to the agent used, the clinical condition of the patient, or other variables (eg, dosages, genetic profile, epigenetic mechanisms). An ongoing study is investigating potential correlations between the response to OXN and clinical characteristics such as the type of pain, psychological structure, comorbidities, and cotreatments to better understand the determinants and degree of analgesic response.

\section{Acknowledgments}

The study was completed independently with no funding. English language editing assistance was provided by Nicola Ryan, on behalf of HPS Italy and funded by Mundipharma Pharmaceuticals srl, Italy.

\section{Disclosure}

The authors report no conflicts of interest in this work.

\section{References}

1. World Health Organization. Cancer Pain Relief: With a Guide to Opioid Availability. Geneva: World Health Organization; 1996.

2. Caraceni A, Hanks G, Kaasa S, et al; European Palliative Care Research Collaborative (EPCRC); European Association for Palliative Care (EAPC). Use of opioid analgesics in the treatment of cancer pain: evidence-based recommendations from the EAPC. Lancet Oncol. 2012;13(2): e58-e68.

3. Wiffen PJ. Evidence-based pain management and palliative care in issue one for 2005 of The Cochrane Library. J Pain Palliat Care Pharmacother. 2005;9(3):65-68.

4. Corli O, Montanari M, Deandrea S, Greco MT, Villani W, Apolone G. An exploratory analysis on the effectiveness of four strong opioids in patients with cancer pain. Pain Med. 2012;13(7):897-907.

5. Caraceni A, Pigni A, Brunelli C. Is oral morphine still the first choice opioid for moderate to severe cancer pain? A systematic review within the European Palliative Care Research Collaborative guidelines project. Palliat Med. 2011;25(5):402-409.

6. King SJ, Reid C, Forbes K, Hanks G. A systematic review of oxycodone in the management of cancer pain. Palliat Med. 2011;25(5):454-470.

7. Mancini I, Bruera E. Constipation in advanced cancer patients. Support Care Cancer. 1998;6(4):356-364.

8. Xiao Y, Liu J, Huang XE, et al. Clinical study on fluvoxamine combined with oxycodone prolonged-release tablets in treating patients with moderate to severe cancer pain. Asian Pac J Cancer Prev. 2014;15(23): 10445-10449.

9. Camilleri M. Opioid-induced constipation: challenges and therapeutic opportunities. Am J Gastroenterol. 2011;106(5):835-842.

10. Wiffen PJ, Derry S, Moore RA. Impact of morphine, fentanyl, oxycodone or codeine on patient consciousness, appetite and thirst when used to treat cancer pain. Cochrane Database Syst Rev. 2014;29(5): CD011056.
11. Weschules DJ, Bain KT, Reifsnyder J, et al. Toward evidence-based prescribing at end of life: a comparative analysis of sustained-release morphine, oxycodone, and transdermal fentanyl, with pain, constipation, and caregiver interaction outcomes in hospice patients. Pain Med. 2006;7(4):320-329.

12. Ripamonti CI, Santini D, Maranzano E, Berti M, Roila F; ESMO Guidelines Working Group. Management of cancer pain: ESMO clinical practice guidelines. Ann Oncol. 2012;23(suppl 7):vii139-vii154.

13. Mundipharma Pharmaceuticals Limited. Targin $5 / 2.5 \mathrm{mg}, 10 \mathrm{mg} / 5 \mathrm{mg}$, $20 \mathrm{mg} / 10 \mathrm{mg}$ and 40/20 mg Prolonged Release Tablets: Summary of Product Characteristics. 2013. Available from: http://www.medicines. ie/medicine/14383/SPC/Targin+10mg+5mg+and+20mg+10mg+prolo nged+release+tablets/. Accessed 15 Jul 2014.

14. Smith K, Hopp M, Mundin G, et al. Low absolute bioavailability of oral naloxone in healthy subjects. Int J Clin Pharmacol Ther. 2012;50(5): 360-367.

15. Poelaert J, Koopmans-Klein G, Dioh A, et al. Treatment with prolongedrelease oxycodone/naloxone improves pain relief and opioid-induced constipation compared with prolonged-release oxycodone in patients with chronic severe pain and laxative-refractory constipation. Clin Ther. 2015;37(4):784-792.

16. Koopmans G, Simpson K, De Andrés J, Lux EA, Wagemans M, Van Megen Y. Fixed ratio (2:1) prolonged-release oxycodone/ naloxone combination improves bowel function in patients with moderate-to-severe pain and opioid-induced constipation refractory to at least two classes of laxatives. Curr Med Res Opin. 2014;30(11): 2389-2396.

17. Burness CB, Keating GM. Oxycodone/Naloxone prolonged-release: a review of its use in the management of chronic pain while counteracting opioid-induced constipation. Drugs. 2014;74(3):401-402.

18. Vondrackova D, Leyendecker P, Meissner W, et al. Analgesic efficacy and safety of oxycodone in combination with naloxone as prolonged release tablets in patient with moderate to severe chronic pain. J Pain. 2008;9:1144-1154.

19. Simpson K, Leyendecker P, Hopp M, et al. Fixed-ratio combination oxycodone/naloxone compared with oxycodone alone for the relief of opioid-induced constipation in moderate-to-severe non-cancer pain. Curr Med Res Opin. 2008;24:3503-3512.

20. Lowenstein O, Leyendecker P, Hopp M, et al. Combined prolongedrelease oxycodone and naloxone improves bowel function in patients receiving opioids for moderate-to-severe non-malignant chronic pain - a randomized controlled trial. Expert Opin Pharmacother. 2009;10:531-543.

21. Cuomo A, Russo G, Esposito G, Forte CA, Connola M, Marcassa C. Efficacy and gastrointestinal tolerability of oral oxycodone/naloxone combination for chronic pain in outpatients with cancer: an observational study. Am J Hosp Palliat Care. 2014;31(8):867-876.

22. Clemens KE, Quednau I, Klaschik E. Bowel function during pain therapy with oxycodone/naloxone prolonged-release tablets in patients with advanced cancer. Int J Clin Pract. 2011;65(4):472-478.

23. Schutter U, Grunert S, Meyer C, Schmidt T, Nolte T. Innovative pain therapy with a fixed combination of prolonged-release oxycodone/ naloxone: a large observational study under conditions of daily practice. Curr Med Res Opin. 2010;26(6):1377-1387.

24. Ahmedzai SH, Nauck F, Bar-Sela G, Bosse B, Leyendecker P, Hopp M. A randomized, double-blind, active-controlled, double-dummy, parallel-group study to determine the safety and efficacy of oxycodone/ naloxone prolonged-release tablets in patients with moderate/severe, chronic cancer pain. Palliat Med. 2012;26(1):50-60.

25. Ahmedzai SH, Leppert W, Janecki M, et al. Long-term safety and efficacy of oxycodone/naloxone prolonged-release tablets in patients with moderate-to-severe chronic cancer pain. Support Care Cancer. 2015;23(3):823-830.

26. Apolone G, Deandrea S, Montanari M, Corli O, Greco MT, Cavuto S. Evaluation of the comparative analgesic effectiveness of transdermal and oral opioids in cancer patients: a propensity score analysis. Eur $J$ Pain. 2012;16(2):229-238. 
27. Rosenbaum PR, Rubin DB. The central role of the propensity score in observational studies for causal effects. Biometrika. 1983;70:41-55.

28. Kaasa S, Apolone G, Klepstad P, et al. European Palliative Care Research Collaborative (EPCRC); European Association for Palliative Care Research Network (EAPCRN). Expert conference on cancer pain assessment and classification. The need for international consensus: working proposals on international standards. BMJ Support Palliat Care. 2011;1(3):281-287.

29. Corli O, Montanari M, Greco MT, et al. How to evaluate the effect of pain treatments in cancer patients: results from a longitudinal outcomes and endpoint Italian cohort study. Eur J Pain. 2013;17(6):858-866.

30. Bennett MI, Attal N, Backonja MM, et al. Using screening tools to identify neuropathic pain. Pain. 2007;127:199-203.

31. Bouhassira D, Attal N, Fermanian J, et al. Development and validation of the Neuropathic Pain Symptom Inventory. Pain. 2004;108:248-257.

32. Mercadante S, Fulfaro F, Casuccio A, Barresi L. Investigation of an opioid response categorization in advanced cancer patients. $J$ Pain Symptom Manage. 1999;18(5):347-352.

33. Kosinski M, Janagap CC, Gajria K, Schein J. Psychometric testing and validation of the Chronic Pain Sleep Inventory. Clin Ther. 2007; 29(suppl):2562-2577.

34. Ware J, Kosinski M, Keller SD. A 12-item short-form health survey: construction of scales and preliminary tests of reliability and validity. Med Care. 1996;34:220-233.

35. Stewart AL, Greenfield S, Hays RD, et al. Functional status and wellbeing of patients with chronic conditions. Results from the Medical Outcomes Study. JAMA. 1989;262:907-913.

36. Rentz AM, Yu R, Muller-Lissner S, Leyendecker P. Validation of the bowel function index to detect clinically meaningful changes in opioidinduced constipation. J Med Econ. 2009;12(4):371-383.

37. Rentz AM, van Hanswijck de Jonge P, Leyendecker P, Hopp M. Observational, nonintervention, multicenter study for validation of the bowel function index for constipation in European countries. Curr Med Res Opin. 2011;27(1):35-44.
38. Cavuto S, Bravi F, Grassi MC, Apolone G. Propensity score for the analysis of observational data: an introduction and an illustrative example. Drug Dev Res. 2006;67:208-216.

39. Brookhart MA, Schneeweiss S, Rothman KJ, Glynn RJ, Avorn J, Stürmer T. Variable selection for propensity score models. Am J Epidemiol. 2006;163(12):1149-1156.

40. Ho DE, Imai K, King G, Stuart EA. MatchIt: nonparametric preprocessing for parametric causal inference. $J$ Stat Soft. 2011;42(8):1-28.

41. Uberall MA, Mueller-Schwefe GH. Safety and efficacy of oxycodone/ naloxone vs oxycodone vs morphine for the treatment of chronic low back pain: results of a 12-week prospective, randomized, open-label blinded endpoint streamlined study with Prolonged-Release preparations. Curr Med Res Opin. 2015;5:1-51.

42. Dworkin RH, Turk DC, Wyrwich KW, et al. Interpreting the clinical importance of treatment outcomes in chronic pain clinical trials: IMMPACT recommendations. J Pain. 2008;9(2):105-121.

43. Farrar JT, Portenoy RK, Berlin JA, Kinman JL, Strom BL. Defining the clinically important difference in pain outcome measures. Pain. 2000;88(3):287-294.

44. Farrar JT, Pritchett YL, Robinson M, Prakash A, Chappell A. The clinical importance of changes in the 0 to 10 numeric rating scale for worst, least, and average pain intensity: analyses of data from clinical trials of duloxetine in pain disorders. J Pain. 2010;11(2):109-118.

45. Farrar JT, Young JP Jr, LaMoreaux L, Werth JL, Poole RM. Clinical importance of changes in chronic pain intensity measured on an 11-point numerical pain rating scale. Pain. 2001;94(2):149-158.

46. Corli O, Roberto A, Greco MT, Montanari M. Assessing the response to opioids in cancer patients: a methodological proposal and the results. Supp Care Cancer. 2015;23:1867-1873.
Drug Design, Development and Therapy

\section{Publish your work in this journal}

Drug Design, Development and Therapy is an international, peerreviewed open-access journal that spans the spectrum of drug design and development through to clinical applications. Clinical outcomes, patient safety, and programs for the development and effective, safe, and sustained use of medicines are a feature of the journal, which

\section{Dovepress}

has also been accepted for indexing on PubMed Central. The manuscript management system is completely online and includes a very quick and fair peer-review system, which is all easy to use. Visit http://www.dovepress.com/testimonials.php to read real quotes from published authors. 